SLAC-PUB-8168

MAY 1999

\title{
LIGHT-FRONT-QUANTIZED QCD IN COVARIANT GAUGE 同
}

\author{
Prem P. Srivastavali \\ and \\ Stanley J. Brodsky \\ Stanford Linear Accelerator Center, Stanford University, Stanford, California 94309
}

\begin{abstract}
The light-front (LF) canonical quantization of quantum chromodynamics in covariant gauge is discussed. The Dirac procedure is used to eliminate the constraints in the gaugefixed front form theory quantum action and to construct the LF Hamiltonian formulation. The physical degrees of freedom emerge naturally. The propagator of the dynamical $\psi_{+}$part of the free fermionic propagator in the LF quantized field theory is shown to be causal and not to contain instantaneous terms. Since the relevant propagators in the covariant gauge formulation are causal, rotational invariance -including the Coulomb potential in the static limit - can be recovered, avoiding the difficulties encountered in light-cone gauge. The Wick rotation may also be performed allowing the conversion of momentum space integrals into Euclidean space forms. Some explicit computations are done in quantum electrodynamics to illustrate the equivalence of front form theory with the conventional covariant formulation. LF quantization thus provides a consistent formulation of gauge theory, despite the fact that the hyperplanes $x^{ \pm}=0$ used to impose boundary conditions constitute characteristic surfaces of a hyperbolic partial differential equation.
\end{abstract}

(Submitted to Physical Review D.)

\footnotetext{
*Research partially supported by the Department of Energy under contract DE-AC03-76SF00515

${ }^{\dagger}$ E-mail: prem@slac.stanford.edu or prem@cbpfsu1.cat.cbpf.br. On leave of absence from Instituto de Física, UERJ-Universidade do Estado de Rio de Janeiro, Brasil.

$\ddagger$ E-mail: sjbth@slac.stanford.edu
} 


\section{Introduction}

The quantization of relativistic field theory at fixed light-front time $\tau=(t-z / c) / \sqrt{2}$, which was proposed by Dirac [1] half a century ago, has found important applications [2, 3, [4 in gauge theory and string theory [5]. The light-front (LF) quantization of QCD in its Hamiltonian form provides an alternative approach to lattice gauge theory for the computation of nonperturbative quantities, such as the spectrum and the light-cone Fock state wavefunctions of relativistic bound states. LF variables have found natural applications in several context, for example, in the quantization of (super-) string theory and M-theory [5]. It has also been employed in the nonabelian bosonization [6] of the field theory of $N$ free Majorana fermions.

Since LF coordinates are not related to the conventional coordinates by a finite Lorentz transformation, the descriptions of the same physical result may be different in the equal-time (instant form) and equal LF-time (front form) of treatments of the theory. This was also found to be the case in the recent study [7] of some soluble twodimensional gauge theory models, where it was also demonstrated that LF quantization is very economical in displaying the relevant degrees of freedom, leading directly to a physical Hilbert space. LF-time-ordered perturbation theory has also been applied [8, 9] to massive fields. It was used in the analysis of the evolution of deep inelastic structure functions [10], the evolution of the distribution amplitudes which control hard exclusive processes in structure functions [10], and the evolution of the distribution amplitudes which control hard exclusive processes in QCD [11]. LF-time-ordered perturbation theory is much more economical than equal-time-ordered perturbation theory, since only graphs with particles with positive LF momenta $p^{+}=\left(p^{0}+p^{3}\right) / \sqrt{2}$ appear. It has been conventional to apply light-front quantization to gauge theory in light cone gauge $A^{+}=\left(A^{0}+A^{3}\right) / \sqrt{2}=0$, since the transverse degrees of freedom of the gauge field can be immediately identified as the dynamical degrees of freedom, and the ghost fields can be ignored in the quantum action of the nonabelian gauge theory [12. However, it does bring severe penalties, such as the breaking of manifest rotational invariance, cumbersome LF time instantaneous interactions, an analytically complex gauge-field propagator, and a difficult renormalization procedure [13]. In addition, light-cone gauge complicates obtaining the Coulomb limit when one applies the LF formalism to simple nonrelativistic systems.

In this paper we will discuss the LF quantization of (massless) gauge field theory in covariant gauges such as Feynman gauge. The penalty in reintroducing FaddeevPopov ghosts and other fields with ghost-like metric is compensated by the restoration

of the Lorentz symmetries. Our general procedure will be first to study the gauge-fixed 
quantum action of the theory on the LF. The self-consistent Hamiltonian formulation is constructed following the Dirac method [14] for constrained systems. It is worth remarking that even after the addition of gauge-fixing and ghost terms in the front form theory, several second class constraints in general remain in the theory. They can be eliminated by constructing the Dirac brackets, and the theory can be quantized canonically by the correspondence principle in terms of a reduced number of independent fields. The commutation relations among them are also found by the Dirac method, and they are useful in order to obtain the momentum space expansions of the fields. The nondynamical projection of the fermion field will be eliminated using a nonlocal constraint equation. The Dyson-Wick perturbation theory expansion [15 based on LF coordinates then becomes straightforward [9]. In fact, the Feynman gauge has been already used [16] in QED in the framework of Weinberg's time-ordered perturbation theory in the infinite-momentum-frame, where it was used to renormalize and compute the order $\alpha, \alpha^{2}$ and part of the $\alpha^{3}$ radiative corrections to the electron anomalous moment. (In this gauge the theory on the LF resembles that of an interacting theory of four scalar fields $\left(A^{+}, A^{\perp}, A^{-}\right)$with the matter fields. The covariant gauge thus should be feasible within the LF framework.) The challenge of renormalization in light-cone gauge and some of its aspects in Feynman gauge were addressed in [17. The front form theory has recently been used [18] to quantize a nuclear physics model in which massive vector mesons couple to field-theoretic nucleons. In this approach, a canonical transformation [8, 9] of the dynamical nucleon $\psi_{+}$field is used to simplify the constraint equation for $\psi_{-}$(in abelian theory). The inclusion of fields with ghost-like metric in discretized light-cone quantization (DLCQ) 19] has been discussed in [20].

The propagator for the dynamical component $\psi_{+}(x)$ of the free fermionic field on the LF is shown to be causal, and it is demonstrated that, in the context of LF-timeordered Dyson-Wick perturbation theory, it has no instantaneous term. This effect [11] is replaced now by explicit seagull and other interaction terms which can be evaluated systematically. Such interactions can be incorporated into a nonperturbative approach such as DLCQ. Next QCD is canonically quantized on the LF in covariant Feynman (or Landau) gauge. A novel feature of the gauge theory interactions in covariant gauges on the LF is the off-diagonal couplings of the $A^{+}$and $A^{-}$fields. Some illustrations are given on the application the Dyson-Wick perturbation theory expansion based on the Wick theorem, built on the LF-time-ordered product. We recall that it was also used [21] to renormalize two-dimensional scalar field theory on the LF, with nonlocal interaction. It was also shown there [21] that even on the LF one may perform the Wick rotation in momentum space integrals and go over to the Euclidean-space integrals, rendering the computation of high order corrections as straightforward as in conventional theory. 


\section{Notation}

The Lagrangian density corresponding to the quantum action [22] of QCD is described in standard notation by

$$
\mathcal{L}_{Q C D}=-\frac{1}{4} F^{a \mu \nu} F_{\mu \nu}^{a}+B^{a} \partial_{\mu} A^{a \mu}+\frac{\xi}{2} B^{a} B^{a}+i \partial^{\mu} \chi_{1}{ }^{a} \mathcal{D}^{a c}{ }_{\mu} \chi_{2}{ }^{c}+\bar{\psi}^{i}\left(i \gamma^{\mu} D^{i j}{ }_{\mu}-m \delta^{i j}\right) \psi^{j}
$$

Here $\psi^{j}$ is the quark field with color index $j=1 . . N_{c}$ for an $S U\left(N_{c}\right)$ color group, $A^{a}{ }_{\mu}$ the gluon field, $F_{\mu \nu}^{a}=\partial_{\mu} A_{\nu}^{a}-\partial_{\nu} A^{a}{ }_{\mu}+g f^{a b c} A^{b}{ }_{\mu} A^{c}{ }_{\nu}$ the field strength, $\mathcal{D}^{a c}{ }_{\mu}=\left(\delta^{a c} \partial_{\mu}+\right.$ $\left.g f^{a b c} A^{b}{ }_{\mu}\right), D^{i j}{ }_{\mu} \psi^{j}=\left(\delta^{i j} \partial_{\mu}-i g A^{a}{ }_{\mu}\left(\lambda^{a} / 2\right)^{i j}\right) \psi^{j}, a=1 . .\left(N_{c}^{2}-1\right)$ the gauge group index etc. The covariant gauge-fixing is introduced by adding to the Lagrangian the linear gauge-fixing term $\left(B^{a} \partial_{\mu} A^{a \mu}+\frac{\xi}{2} B^{a} B^{a}\right)$ where $B^{a}$ is the Nakanishi-Lautrup auxiliary field and $\xi$ is a parameter. The $\chi_{1}{ }^{a}$ and $\chi_{2}{ }^{a}$ are the (hermitian) anticommuting FaddeevPopov ghost fields, and the action is invariant under the BRS [23] transformation.

The LF coordinates $\mu=+,-, 1,2$ are defined as $x^{\mu}=\left(x^{+}=x_{-}=\left(x^{0}+\right.\right.$ $\left.\left.x^{3}\right) / \sqrt{2}, x^{-}=x_{+}=\left(x^{0}-x^{3}\right) / \sqrt{2}, x^{\perp}\right)$ where $x^{\perp}=\left(x^{1}, x^{2}\right)=\left(-x_{1},-x_{2}\right)$ are the transverse coordinates and $\mu=+,-, 1,2$. The $x^{+} \equiv \tau$ will be taken as the LF-time coordinate while $x^{-}$is the longitudinal spatial one. We can of course make the convention with the role of $x^{+}$and $x^{-}$interchanged. The equal- $x^{+}$quantized theory does seem to already contain the information on the equal- $x^{-}$commutator [7]. For the fourmomentum components we have $k^{\mu}=\left(k^{-} \equiv\left(k^{0}-k^{3}\right) / \sqrt{2}, k^{+} \equiv\left(k^{0}+k^{3}\right) / \sqrt{2}, k^{\perp}\right)$ where $k^{+}$indicates the longitudinal momentum and $k^{-}$the LF energy. The LF components of any tensor, for example, the gauge field $\left(A^{+}, A^{-}, A^{\perp}\right)$ are similarly defined, and the metric tensor $g_{\mu \nu}$ may be read from $A \cdot B=A^{+} B^{-}+A^{-} B^{+}-A^{\perp} B^{\perp}$. The $\gamma^{ \pm}$defined by $\gamma^{ \pm}=\left(\gamma^{0} \pm \gamma^{3}\right) / \sqrt{2}$ satisfy $\left(\gamma^{+}\right)^{2}=\left(\gamma^{-}\right)^{2}=0, \gamma^{0} \gamma^{+}=\gamma^{-} \gamma^{0}, \gamma^{+} \gamma^{-}+\gamma^{-} \gamma^{+}=2 I$, $\gamma^{+\dagger}=\gamma^{-}$and it follows that $\Lambda^{ \pm}=\frac{1}{2} \gamma^{\mp} \gamma^{ \pm}=\frac{1}{\sqrt{2}} \gamma^{0} \gamma^{ \pm}$are hermitian projection operators: $\quad\left(\Lambda^{ \pm}\right)^{2}=\Lambda^{ \pm}, \quad \Lambda^{+} \Lambda^{-}=\Lambda^{-} \Lambda^{+}=0, \quad \gamma^{0} \Lambda^{+}=\Lambda^{-} \gamma^{0}$. The corresponding \pm projections of the LF Dirac spinor are $\psi_{ \pm}=\Lambda^{ \pm} \psi$ and $\psi=\psi_{+}+\psi_{-}, \bar{\psi}=\psi^{\dagger} \gamma^{0}=$ $\bar{\psi}_{+}+\bar{\psi}_{-}, \gamma^{ \pm} \psi_{\mp}=0$ etc.

\section{Spinor field propagator on the LF}

The quark field term in LF coordinates reads

$$
\begin{aligned}
\bar{\psi}^{i}\left(i \gamma^{\mu} D^{i j}{ }_{\mu}-m \delta^{i j}\right) \psi^{j} & =i \sqrt{2} \bar{\psi}_{+}^{i} \gamma^{0} D^{i j}{ }_{+} \psi_{+}{ }^{j}+\bar{\psi}_{+}^{i}\left(i \gamma^{\perp} D^{i j}{ }_{\perp}-m \delta^{i j}\right) \psi_{-}^{j} \\
& +\bar{\psi}_{-}^{i}\left[i \sqrt{2} \gamma^{0} D^{i j}{ }_{-} \psi_{-}{ }^{j}+\left(i \gamma^{\perp} D^{i j}{ }_{\perp}-m \delta^{i j}\right) \psi_{+}{ }^{j}\right]
\end{aligned}
$$


where $D^{i j}{ }_{ \pm}=\left(\delta^{i j} \partial_{ \pm}-i g A^{a}{ }_{ \pm}\left(\lambda^{a} / 2\right)^{i j}\right)$. It shows that the minus components $\psi_{-}^{j}$ are in fact nondynamical ( Lagrange multiplier ) fields without kinetic terms. The variation of the action with respect to $\bar{\psi}^{j}$ - and $\psi^{j}$ - leads to the following gauge covariant constraint equation

$$
i \sqrt{2} D^{i j}{ }_{-} \psi_{-}{ }^{j}=-\left(i \gamma^{0} \gamma^{\perp} D^{i j}{ }_{\perp}-m \gamma^{0} \delta^{i j}\right) \psi_{+}{ }^{j},
$$

and its conjugate. The $\psi^{j}$ - components may thus be eliminated in favor of the dynamical components $\psi_{+}^{j}$

$$
\psi_{-}^{j}(x)=\frac{i}{\sqrt{2}}\left[U^{-1}\left(x \mid A_{-}\right) \frac{1}{\partial_{-}} U\left(x \mid A_{-}\right)\right]^{j k}\left(i \gamma^{0} \gamma^{\perp} D^{k l}{ }_{\perp}-m \gamma^{0} \delta^{k l}\right) \psi_{+}^{l}(x) .
$$

Here, for a fixed $\tau, U \equiv U\left(x \mid A_{-}\right)$is an $N_{c} \times N_{c}$ gauge matrix in the fundamental representation of $S U\left(N_{c}\right)$ and it satisfies

$$
\partial_{-} U\left(x \mid A_{-}\right)=-i g U\left(x \mid A_{-}\right) A_{-}(x)
$$

with $A_{-}=A^{a}{ }_{-} \lambda^{a} / 2$. It has the formal solution

$$
U\left(x^{-}, x^{\perp} \mid A_{-}\right)=U\left(x_{0}^{-}, x^{\perp} \mid A_{-}\right) \tilde{\mathcal{P}} \exp \left\{-i g \int_{x^{-} 0}^{x^{-}} d y^{-} A_{-}\left(y^{-}, x^{\perp}\right)\right\}
$$

where $\tilde{\mathcal{P}}$ indicates the anti-path-ordering along the longitudinal direction $x^{-} . U$ has a series expansion in the powers of the coupling constant.

The free field propagator for $\psi_{+}$is determined from the following quadratic terms in the Lagrangian (suppressing the color index) density

$$
i \sqrt{2} \psi_{+}^{\dagger} \partial_{+} \psi_{+}+\psi_{+}^{\dagger}\left(i \gamma^{0} \gamma^{\perp} \partial_{\perp}-m \gamma^{0}\right) \psi_{-}
$$

contained in (2). Here we have used the free field constraint equation $2 i \partial_{-} \psi_{-}=$ $\left(i \gamma^{\perp} \partial_{\perp}+m\right) \gamma^{+} \psi_{+}$which determines the dependent field $\psi_{-}$. The equation of motion for the independent component $\psi_{+}$is nonlocal in the longitudinal direction

$$
\left[4 \partial_{+}+\left(m+i \gamma^{\perp} \partial_{\perp}\right) \gamma^{-} \frac{1}{\partial_{-}}\left(m+i \gamma^{\perp} \partial_{\perp}\right) \gamma^{+}\right] \psi_{+}=0
$$

The free field Hamiltonian formulation can be constructed by following the Dirac procedure [14]. The constraint equation arises now as a second class constraint on the canonical phase space. The Dirac bracket which takes care of these constraints is easily constructed. The effective free LF Hamiltonian is found to be $\mathcal{H}^{l f}=-\bar{\psi}_{+}\left(i \gamma^{\perp} \partial_{\perp}-m\right) \psi_{-}$ and the canonical quantization performed by the correspondence of the Dirac brackets 
with the (anti-)commutators leads to the following nonvanishing local anticommutation relation

$$
\left\{\psi_{+}\left(\tau, x^{-}, x^{\perp}\right), \psi_{+}^{\dagger}\left(\tau, y^{-}, y^{\perp}\right)\right\}=\frac{1}{\sqrt{2}} \Lambda^{+} \delta\left(x^{-}-y^{-}\right) \delta^{2}\left(x^{\perp}-y^{\perp}\right) .
$$

on the LF. They were proposed earlier in Ref. [8]. The equation of motion (8) for $\psi_{+}$ is recovered as an Heisenberg equation of motion if we employ (9).

The propagator in momentum space may be derived by going over to the Fourier transform of $\psi(x)$ over the complete set of linearly independent plane wave solutions of the free Dirac equation, say, for $p^{+}>0$. Such a set is spanned by $u^{(r)}(p) e^{-i p \cdot x}$ together with $v^{(r)}(p) e^{i p \cdot x}$ where $u^{(r)}(p)$ and $v^{(r)}(p) \equiv u^{(r)}(p)_{c}=C \gamma^{0^{T}} u^{(r)}(p)^{*}$ are linearly independent solutions of the Dirac equation in momentum space: $\left(\gamma^{\mu} p_{\mu}-m\right) u^{(r)}(p)=0$ and $p \cdot x=\left(p^{-} x^{+}+p^{+} x^{-}-p^{\perp} x^{\perp}\right)$.

A useful form [24] of the solution for the four-spinors in the context of the LF quantization is

$$
u^{(r)}(p)=\frac{1}{\left(\sqrt{2} p^{+} m\right)^{\frac{1}{2}}}\left[\sqrt{2} p^{+} \Lambda^{+}+\left(m+\gamma^{\perp} p_{\perp}\right) \Lambda^{-}\right] \tilde{u}^{(r)}
$$

where the constant spinors $\tilde{u}^{(r)}$ satisfy $\gamma^{0} \tilde{u}^{(r)}=\tilde{u}^{(r)}$ and $\Sigma_{3} \tilde{u}^{(r)}=r \tilde{u}^{(r)}$ with $\Sigma_{3}=i \gamma^{1} \gamma^{2}$ and $r= \pm$. The normalization and the completeness relations are: $\bar{u}^{(r)}(p) u^{(s)}(p)=$ $\delta_{r s}=-\bar{v}^{(r)}(p) v^{(s)}(p), \quad \sum_{r= \pm} u^{(r)}(p) \bar{u}^{(r)}(p)=(\not p+m) / 2 m, \quad \sum_{r= \pm} v^{(r)}(p) \bar{v}^{(r)}(p)=$ $(\not p-m) / 2 m$ and $\mathrm{C}$ is the charge conjugation matrix 15.

Its $\Lambda^{+}$projection is by construction very simple, $u^{(r)}{ }_{+}(p)=\left(\sqrt{2} p^{+} / m\right)^{\frac{1}{2}}\left(\Lambda^{+} \tilde{u}^{(r)}\right)$ and they are eigenstates of $\Sigma_{3}$ as well while the $\tilde{u}^{(r)}$ correspond to the rest frame spinors for which $\sqrt{2} p^{ \pm}=m$.

The Fourier transform expansion may be written as

$$
\psi(x)=\frac{1}{\sqrt{(2 \pi)^{3}}} \sum_{r= \pm} \int d^{2} p^{\perp} d p^{+} \theta\left(p^{+}\right) \sqrt{\frac{m}{p^{+}}}\left[b^{(r)}(p) u^{(r)}(p) e^{-i p . x}+d^{\dagger(r)}(p) v^{(r)}(p) e^{i p . x}\right]
$$

The presence of the factor $\theta\left(p^{+}\right)$in (11) follows also from the considerations of the covariant phase space (or LIPS) factor which is found relevant 25] in the context of the analysis of the physical processes. For $\psi_{+} \equiv \Lambda^{+} \psi$ we find

$$
\psi_{+}(x)=\frac{(\sqrt{2})^{\frac{1}{2}}}{\sqrt{(2 \pi)^{3}}} \sum_{r= \pm} \int d^{2} p^{\perp} d p^{+} \theta\left(p^{+}\right)\left[b^{(r)}(p) \tilde{u}_{+}^{(r)} e^{-i p . x}+d^{\dagger(r)}(p) \tilde{v}_{+}^{(r)} e^{i p . x}\right],
$$

where $\tilde{u}$ and $\tilde{v}$ are constant spinors, and the integrations are to be taken from $-\infty$ to $\infty$ not only for $p^{\perp}$ but also over $p^{+}$. It can be verified that the anticommutation 
relations (9) are satisfied if the creation and the annihilation operators are assumed to satisfy the canonical anticommutation relations, with the nonvanishing ones given by: $\left\{b^{(r)}(p), b^{\dagger(s)}\left(p^{\prime}\right)\right\}=\left\{d^{(r)}(p), d^{\dagger(s)}\left(p^{\prime}\right)\right\}=\delta_{r s} \delta\left(p^{+}-p^{\prime+}\right) \delta^{2}\left(p^{\perp}-p^{\prime \perp}\right)$.

The free propagator for the independent component $\psi_{+}$is easily derived using (12) and (10) and it is given by

$$
\begin{aligned}
\left\langle 0\left|T\left(\psi_{+A}(x) \psi_{+B}^{\dagger}(0)\right)\right| 0\right\rangle & =\left\langle 0\left|\left[\theta(\tau) \psi_{+A}(x) \psi_{+B}^{\dagger}(0)-\theta(-\tau) \psi_{+B}^{\dagger}(0) \psi_{+A}(x)\right]\right| 0\right\rangle \\
& =\frac{1}{\sqrt{2}} \frac{\Lambda_{A B}^{+}}{(2 \pi)^{3}} \int d^{2} q^{\perp} d q^{+} \theta\left(q^{+}\right)\left[\theta(\tau) e^{-i q x}-\theta(-\tau) e^{i q x}\right](13)
\end{aligned}
$$

where $A, B=1.4$ label the spinor components and we have used (12). The only relevant differences, compared with the case of the scalar field, are, apart from the appearance of the projection operator, the absence of the factor $\left(1 / 2 q^{+}\right)$in the integrand of $(13)$ and the negative sign of the second term in the fermionic case. They, however, compensate, and the standard manipulations to factor out the exponential give rise to the factor $\left[\theta\left(q^{+}\right)+\theta\left(-q^{+}\right)\right]$which may be interpreted as unity in the distribution theory sense, parallel to what we find in the derivation of the scalar field propagator on the LF. The straightforward use of the integral representation $2 \pi i \theta(\tau) e^{-i p \tau}=\int d \lambda e^{(-i \lambda \tau)} /(p-\lambda-$ $i \epsilon)$ of $\theta( \pm \tau)$, together with the standard manipulations in the second term to factor out the exponential, leads to

$$
<0\left|T\left(\psi_{+}^{i}(x) \psi_{+}^{\dagger j}(0)\right)\right| 0>=\frac{i \delta^{i j}}{(2 \pi)^{4}} \int d^{4} q \frac{\sqrt{2} q^{+} \Lambda^{+}}{\left(q^{2}-m^{2}+i \epsilon\right)} e^{-i q \cdot x},
$$

where for convenience we have renamed the dummy integration variable originating from the integral representation of $\theta( \pm \tau)$ as $q^{-}$and $d^{4} q=d^{2} q^{\perp} d q^{+} d q^{-}$with all integrations ranging from $-\infty$ to $\infty$. It may also be derived straightforwardly by functional integral; we do have to take care of the second class constraint in the measure. We have restored the color index as well. The propagator (14) contains no instantaneous term [8], and the integrand factor may also be expressed as $\approx\left[\Lambda^{+}(\not q+m) \Lambda^{-} /\left(q^{2}-m^{2}+i \epsilon\right)\right] \gamma^{0}$. We verify that the propagator satisfies the equation for the Green's function corresponding to the equation of motion of $\psi_{+},(8)$.

\section{Gauge field propagator}

We now turn our attention to the gauge field propagator on the LF in the covariant gauges. The relevant quadratic terms in the Lagrangian density are

$$
\frac{1}{2}\left[F^{a}{ }_{+-} F^{a}+-+2 F^{a}{ }_{+\perp} F_{-\perp}^{a}-F_{12}^{a} F^{a}{ }_{12}\right]++B^{a} \partial_{\mu} A^{a \mu}+\frac{\xi}{2} B^{a} B^{a}+i \partial^{\mu} \chi_{1}{ }^{a} \partial_{\mu} \chi_{2}{ }^{a}
$$


It will be sufficient to study the free abelian gauge theory described by the following Lagrangian density

$$
\frac{1}{2}\left[\left(F_{+-}\right)^{2}-\left(F_{12}\right)^{2}+2 F_{+\perp} F_{-\perp}\right]+B\left(\partial_{+} A_{-}+\partial_{-} A_{+}+\partial_{\perp} A^{\perp}\right)+\frac{\xi}{2} B^{2}
$$

where for the abelian theory $F_{\mu \nu} \equiv\left(\partial_{\mu} A_{\nu}-\partial_{\nu} A_{\mu}\right)$. The canonical momenta are $\pi^{+}=0$, $\pi_{B}=0, \pi^{\perp}=F_{-\perp}, \pi^{-}=F_{+-}+B$ and the canonical Hamiltonian density is found to be

$$
\mathcal{H}_{c}=\frac{1}{2}\left(\pi^{-}\right)^{2}+\frac{1}{2}\left(F_{12}\right)^{2}-A_{+}\left(\partial_{-} \pi^{-}+\partial_{\perp} \pi^{\perp}-2 \partial_{-} B\right)-B\left(\pi^{-}+\partial_{\perp} A^{\perp}\right)+\frac{1}{2}(1-\xi) B^{2}
$$

In the Dirac procedure the primary constraints [14 are $\pi^{+} \approx 0, \pi_{B} \approx 0$ and $\eta \equiv$ $\pi^{\perp}-\partial_{-} A_{\perp}+\partial_{\perp} A_{-} \approx 0$, where $\perp=1,2$ and $\approx$ stands for weak equality relation [14]. We now require the persistency in $\tau$ of these constraints employing the preliminary Hamiltonian, which is obtained by adding to the canonical Hamiltonian the primary constraints multiplied by the Lagrange multiplier fields. We assume the standard Poisson brackets for the dynamical variables in the computation for obtaining the Hamilton's equations of motion. We are led to the following two secondary constraints

$$
\begin{aligned}
\Phi \equiv \partial_{-} \pi^{-}+\partial_{\perp} \pi^{\perp}-2 \partial_{-} B & \approx 0 \\
\Psi \equiv \pi^{-}+2 \partial_{-} A_{+}+\partial_{\perp} A^{\perp}-(1-\xi) B & \approx 0 .
\end{aligned}
$$

The Hamiltonian is next enlarged by including these additional constraints as well. The procedure is repeated. No more constraints are seen to arise, and we are left only with the equations which would determine the Lagrange multiplier fields. According to the Dirac procedure [14], we now go over from the standard Poisson brackets to the modified Poisson brackets, called frequently Dirac brackets, such that inside them we are able to substitute the above constraints as strong equality relations (e.g., by = in place of $\approx)$. The equal- $\tau$ Dirac bracket $\{f(x), g(y)\}_{D}$ which carries this property is constructed straightforwardly. Hamilton's equations now employ the Dirac brackets and the phase space constraints $\pi^{+}=0, \pi_{B}=0, \eta=0, \Phi=0$, and $\Psi=0$ then effectively reduce the (extended) Hamiltonian. In the covariant Feynman gauge with $\xi=1$ the free Hamiltonian takes the simple form

$$
H_{0}{ }^{L F}=-\frac{1}{2} \int d^{2} x^{\perp} d x^{-} g^{\mu \nu} A_{\mu} \partial^{\perp} \partial_{\perp} A_{\nu} .
$$

The theory is canonically quantized through the correspondence $i\{f(x), g(y)\}_{D} \rightarrow$ $[f(x), g(y)]$, the commutator among the corresponding operators. The equal- $\tau$ commutators of the gauge field are found to be $\left[A_{\mu}(x), A_{\nu}(y)\right]_{x^{+}=y^{+}=\tau}=-i g_{\mu \nu} K(x, y)$ 
where $K(x, y)=-(1 / 4) \epsilon\left(x^{-}-y^{-}\right) \delta\left(x^{\perp}-y^{\perp}\right)$ is nonlocal in the longitudinal coordinate. The transverse components of the gauge field have the physical LF commutators $\left[A_{\perp}(x), A_{\perp^{\prime}}(y)\right]_{\tau}=i \delta_{\perp, \perp^{\prime}} K(x, y)$, while for the \pm components we have only the mixed commutator nonvanishing $\left[A_{+}(x), A_{-}(y)\right]_{\tau}=-i K(x, y)$, it has a negative sign which indicates the presence of unphysical degrees of freedom in Feynman gauge. For $\xi \neq 1$ the commutator, for example, of $A_{ \pm}$with $A_{\perp}$ is found to be nonvanishing.

The Heisenberg equations of motion lead to $\square A_{\mu}=0$ for all the components, and consequently the Fourier transform of the free gauge field over the complete set of plane wave solutions takes the following form on the LF

$$
A^{\mu}(x)=\frac{1}{\sqrt{(2 \pi)^{3}}} \int d^{2} k^{\perp} d k^{+} \frac{\theta\left(k^{+}\right)}{\sqrt{2 k^{+}}} e^{\mu(\lambda)}(k)\left[a_{(\lambda)}\left(k^{+}, k^{\perp}\right) e^{-i k \cdot x}+a_{(\lambda)}^{\dagger}\left(k^{+}, k^{\perp}\right) e^{i k \cdot x}\right]
$$

where $e^{\mu(\lambda)}(k), \lambda=-,+, 1,2$ label the set of four linearly independent polarization four-vectors. In the front form theory the two transverse (physical) polarization vector are space-like while the others are null four-vectors. For a fixed $k^{\mu}=\left(k^{0}, \vec{k}\right)$, where $k^{0}=$ $|\vec{k}|$, we may construct them as follows: $e^{(+)}=\left(1, \vec{k} / k^{0}\right) / \sqrt{2}, e^{(-)}=\left(1,-\vec{k} / k^{0}\right) / \sqrt{2}$, $e^{(1)}=(0, \vec{\epsilon}(k ; 1))$, and $e^{(2)}=(0, \vec{\epsilon}(k ; 2))$. Here $(0,1,2,3)$ components are specified for convenience while $\vec{\epsilon}(k ; 1), \vec{\epsilon}(k ; 2)$ and $\vec{k} /|\vec{k}|$ constitute the usual orthonormal set of 3 -vectors. The polarization vectors are orthonormal: $g_{\mu \nu} e^{(\lambda) \mu}(k) e^{(\sigma) \nu}(k)=g^{\lambda \sigma}$ and satisfy the completeness relation: $g_{\lambda \sigma} e^{(\lambda)}{ }_{\mu}(k) e^{(\sigma)}{ }_{\nu}(k)=g_{\mu \nu}$. The field commutation relations for the gauge field found above are shown to be satisfied if we assume, parallel to the discussion in the fermionic case, the canonical commutation relations: $\left[a_{(\lambda)}\left(k^{+}, k^{\perp}\right), a_{(\sigma)}^{\dagger}\left(k^{\prime+}, k^{\perp}\right)\right]=-g_{\lambda \sigma} \delta\left(k^{+}-k^{\prime+}\right) \delta^{2}\left(k^{\perp}-k^{\prime \perp}\right)$. We note that the operators $a_{(0)}=\left(a_{(+)}+a_{(-)}\right) / \sqrt{2}$ and $a_{(3)}=\left(a_{(+)}-a_{(-)}\right) / \sqrt{2}$ obey the usual canonical commutation relations except that in the case of $a_{(0)}$ a negative sign is obtained. The discussion of the Gupta-Bleuler consistency condition then becomes parallel to that in the conventional equal-time treatment [15] of the theory. The Feynman gauge free gauge field propagator on the LF can be derived straightforwardly using (20)

$$
<0\left|T\left(A^{a}{ }_{\mu}(x) A^{b}{ }_{\nu}(0)\right)\right| 0>=\frac{i \delta^{a b}}{(2 \pi)^{4}} \int d^{4} k e^{-i k \cdot x} \frac{-g_{\mu \nu}}{k^{2}+i \epsilon}
$$

where we have restored the gauge index $a$ and used $\left[a_{(\lambda) a}\left(k^{+}, k^{\perp}\right), a^{\dagger}{ }_{(\sigma) b}\left(k^{\prime+}, k^{\perp}\right)\right]$ $=-g_{\lambda \sigma} \delta_{a b} \delta\left(k^{+}-k^{\prime+}\right) \delta^{2}\left(k^{\perp}-k^{\perp}\right)$. The free ghost-antighost propagators for the anticommuting ghost fields may likewise be derived; they are also causal and given by $\left(i /(2 \pi)^{4}\right) \delta_{a b} /\left(k^{2}+i \epsilon\right)$.) 


\section{$5 \quad$ Illustrations}

The Hamiltonian density in Feynman gauge is

$$
\begin{aligned}
\mathcal{H}^{L F} & =\mathcal{H}_{0}+\mathcal{H}_{i n t} \\
& =-\frac{1}{2} g^{\mu \nu} A^{a}{ }_{\mu} \partial^{\perp} \partial_{\perp} A^{a}{ }_{\nu}-g \sqrt{2} \bar{\psi}_{+}^{i} \gamma^{0} A_{+}{ }^{i j} \psi_{+}{ }^{j} \\
& -\bar{\psi}_{+}^{i}\left[\delta^{i j}\left(i \gamma^{\perp} \partial_{\perp}-m\right)+g \gamma^{\perp} A^{i j}{ }_{\perp}\right] \psi_{-}{ }^{j}+\frac{g}{2} f^{a b c}\left(\partial_{\mu} A^{a}{ }_{\nu}-\partial_{\nu} A^{a}{ }_{\mu}\right) A^{b \mu} A^{c \nu} \\
& +\frac{g^{2}}{4} f^{a b e} f^{c d e} A^{a}{ }_{\mu} A^{b}{ }_{\nu} A^{c \mu} A^{d \nu}+\partial^{\mu}\left(\bar{\chi}^{a}\right) \partial_{\mu} \chi^{a}+g f^{a b c}\left(\partial^{\mu} \bar{\chi}^{a}\right) \chi^{b} A^{c}{ }_{\mu}
\end{aligned}
$$

where $\psi_{-}{ }^{j}$ is given in (4), we have set $\sqrt{2} \chi=\left(\chi_{1}+i \chi_{2}\right), \sqrt{2} \bar{\chi}=\left(\chi_{1}-i \chi_{2}\right)$, and in (22) the cubic and higher order terms belong to $\mathcal{H}_{\text {int }}$ which is also understood to be normal ordered. It is worth remarking that despite the presence of the longitudinal operators $a_{ \pm}$and $a^{\dagger}{ }_{ \pm}$in the fields $A_{\mu}$, there are no non-zero matrix elements involving these quanta as external lines in view of the commmutation relations of these operators as discussed in the previous section.

The perturbation theory expansion in the interaction representation where we time order with respect to the LF time $\tau$ is built following the Dyson-Wick [15] procedure. We will illustrate it in our context through some explicit computations, for simplicity, in QED where $U\left(x \mid A_{-}\right)=\exp \left\{-i e \int^{x^{-}} d u^{-} A_{-}\left(\tau, u^{-}, x^{\perp}\right)\right\}$ and $D_{\mu}=\left(\partial_{\mu}-i e A_{\mu}\right)$. We observe from (4) and (22) that a seagull term of the order $e^{2}$ is present in the interaction Hamiltonian at the tree level as in case of the scalar field QED.

Consider Electron-Muon scattering. The contribution coming from the longitudinal components of the gauge field, viz, $A_{+}$and $A_{-}$, which carry a nonvanishing mixed propagator, is contained in the following normal-ordered product of the Wick decomposition of the second order term in the perturbation theory expansion of the S-matrix

$$
\begin{aligned}
e^{2} \int & d^{4} x_{1} d^{4} x_{2}: \psi^{\dagger}{ }_{e+}\left(x_{1}\right) \psi_{e+}\left(x_{1}\right) \dot{A}_{+}\left(x_{1}\right) \psi_{\mu+}^{\dagger}\left(x_{2}\right)\left(m+i \not \partial^{T}\right) \\
& \frac{1}{2} \times \int d y_{2}{ }^{-} \epsilon\left(x_{2}{ }^{-}-y_{2}{ }^{-}\right)\left\{\int_{y_{2}{ }^{-}}^{x_{2}{ }^{-}} d u_{2}{ }^{-} \dot{A}_{-}\left(u_{2}\right)\right\}\left(m-i \not \partial^{T}\right) \psi_{\mu+}\left(y_{2}\right):
\end{aligned}
$$

where overdots indicate [15 the pair of fields contracted, $x_{2} \equiv\left(\tau_{2}, x_{2}^{-}, x_{2}^{\perp}\right), u_{2} \equiv$ $\left(\tau_{2}, u_{2}^{-}, x_{2}^{\perp}\right), y_{2} \equiv\left(\tau_{2}, y_{2}^{-}, x_{2}^{\perp}\right), x_{1} \equiv\left(\tau_{1}, x_{1}^{-}, x_{1}^{\perp}\right)$, and $\not^{T} \equiv \gamma^{\perp} \partial_{\perp}$. Here we write $\int \epsilon(x-y) d y / 2$ in place of $\partial_{x}{ }^{-1}$ and suppress the symmetry factors for convenience. Making use of the Fourier transforms of the fields and the gauge field propagator the matrix element is written down by simple inspection

$$
e^{2}\left[u_{e+}^{\dagger}\left(p_{1}^{\prime}\right) u_{e+}\left(p_{1}\right) u_{\mu+}^{\dagger}\left(p_{2}^{\prime}\right) \frac{\left(m+\not \not_{2}^{\prime}{ }^{T}\right)}{-i{p^{\prime}}_{2}{ }_{2}} \frac{\left(m-\not p_{2}{ }^{T}\right)}{-i p^{+}{ }_{2}} u_{\mu+}\left(p_{2}\right)\right] \frac{-g_{+-}}{q^{2}+i \epsilon}
$$


where $q^{\mu}=\left(p^{\prime \mu}{ }_{2}-p^{\mu}{ }_{1}\right)$. It may be readily rewritten in view of (10) and the simplifications following from the mass shell conditions on the external lines to give

$$
e^{2}\left[\bar{u}_{e}\left(p_{1}^{\prime}\right) \gamma^{+} u_{e}\left(p_{1}\right) \bar{u}_{\mu}\left(p_{2}^{\prime}\right) \gamma^{-} u_{\mu}\left(p_{2}\right)\right] \frac{-g_{+-}}{q^{2}+i \epsilon}
$$

The graph with the $A_{+}$and $A_{-}$interchanged gives rise to a similar expression with $g_{+-} \rightarrow g_{-+}$while $\gamma^{ \pm} \rightarrow \gamma^{\mp}$. There are four contributions arising from the virtual propagation of the transverse components of the gauge field. They add up to

$$
e^{2}\left[\bar{u}_{e}\left(p_{1}^{\prime}\right) \gamma^{\perp} u_{e}\left(p_{1}\right) \bar{u}_{\mu}\left(p_{2}^{\prime}\right) \gamma^{\perp^{\prime}} u_{\mu}\left(p_{2}\right)\right] \frac{-g_{\perp \perp^{\prime}}}{q^{2}+i \epsilon}
$$

resulting in the following complete matrix element to the second order

$$
e^{2}\left[\bar{u}_{e}\left(p_{1}^{\prime}\right) \gamma^{\mu} u_{e}\left(p_{1}\right) \bar{u}_{\mu}\left(p_{2}^{\prime}\right) \gamma^{\nu} u_{\mu}\left(p_{2}\right)\right] \frac{-g_{\mu \nu}}{q^{2}+i \epsilon} .
$$

which agrees with the one obtained in the conventional equal-time formulation.

Consider next the computation of Electron Self-Energy. The contribution from the longitudinal components arises from

$$
\begin{aligned}
e^{2} \int d^{4} x_{1} d^{4} x_{2} & : \psi_{+}{ }^{\dagger}\left(x_{2}\right)\left(m+i \not \partial_{2}{ }^{T}\right) \int_{-\infty}^{\infty} \frac{1}{2} d y_{2}{ }^{-} \epsilon\left(x_{2}{ }^{-}-y_{2}{ }^{-}\right) \\
& \left\{\int_{y_{2}{ }^{-}}^{x_{2}{ }^{-}} d u_{2}{ }^{-} \dot{A}_{-}\left(u_{2}\right)\right\}\left(m-i \not \partial_{2}{ }^{T}\right) \ddot{\psi}_{+}\left(y_{2}\right) \ddot{\psi}_{+}^{\dagger}\left(x_{1}\right) \psi_{+}\left(x_{1}\right) \dot{A}_{+}\left(x_{1}\right):(28
\end{aligned}
$$

leading to

$$
e^{2} \int d^{4} q \frac{\bar{u}^{(r)}(p)\left[\gamma^{-}\left(m+\not q^{T}\right) \gamma^{+}\right] u^{(s)}(p)}{\left[(p-q)^{2}+i \epsilon\right]\left(q^{2}-m^{2}+i \epsilon\right)}\left(-g_{-+}\right)
$$

The graph with the $A_{+}$and $A_{-}$interchanged gives rise to a similar expression with $g_{+-} \rightarrow g_{-+}$while $\gamma^{ \pm} \rightarrow \gamma^{\mp}$. The matrix elements following from the four graphs corresponding to the exchange of the (photon ) fields $A_{1}$ and $A_{2}$ is also written down by simple inspection. As in the earlier case the expressions get simplified in virtue of (10) and acquire the covariant form encountered in the conventional covariant perturbation theory. The complete matrix element is found to be

$$
e^{2} \int d^{4} q \frac{\bar{u}^{(r)}(p)\left[\gamma^{\mu}(m+\not{q}) \gamma^{\nu}\right] u^{(s)}(p)}{\left[(p-q)^{2}+i \epsilon\right]\left(q^{2}-m^{2}+i \epsilon\right)}\left(-g_{\mu \nu}\right)
$$

where $\tilde{q}^{\mu} \equiv\left(\left(m^{2}+q^{\perp} q^{\perp}\right) /\left(2 q^{+}\right), q^{+}, q^{\perp}\right)$ and the integration measure is $d^{4} q=d^{2} q^{\perp} d q^{+} d q^{-}$. Considering that the integrand has the pole at $q^{2}-m^{2} \approx 0$ we may regard the expression obtained on the LF to be effectively identical to the one obtained in the conventional covariant theory framework. The contribution coming from the seagull term at the tree level vanishes if the dimensional regularization is used. 
The calculation of the tree graphs for Compton scattering, $\gamma+e \rightarrow \gamma+e$, is tedious but straightforward. The results on the LF are shown to be in agreement with the conventional covariant theory one. We remark that on the LF the tree level seagull term dominates the (classical) Thomson formula for the scattering at the vanishingly small photon energies similar to the case of the QED with the scalar fields. In the covariant gauges on the LF all the relevant field propagators in momentum space are causal. Employing (10) and (12) it is rather straightforward to rewrite the final result in manifestly covariant form. The computation to higher orders in the DysonWick perturbation expansion can be carried out straightforwardly; the nonlocality of the interaction, arising from (4), does require some extra effort to handle, but it becomes easier to control the rotational invariance and compute the loop integrals in the traditional fashion.

\section{Conclusions}

The LF Dyson-Wick perturbation theory expansion based on the LF-time-ordering has a number of advantages for computing the high order corrections in the front form $\mathrm{QCD}$ theory. The covariant Feynman (or Landau) gauge may be adopted with advantage on the LF where all the relevant propagators take (except for the numerical projector in the dynamical fermion propagator) the covariant causal forms permitting us to employ the usual power counting rules. The Hamiltonian version [26] can be implemented in DLCQ [19]. In the approximation of no retardation, the Coulomb interaction is recovered. The propagator of the independent component of the fermion field on the LF has been shown to be simpler than in the conventional theory. The momentum space integrals in the front form theory may be converted [21] to the Euclidean space integrals which then permit us to employ, for example, dimensional regularization. The illustrations given here demonstrate the agreement of the LF quantized theory results with the conventional covariant theory ones. The expression (10) for the LF spinor proved to be quite useful here. The fact that in the front form theory the classical Thomson scattering limit is obtained from a seagull term at the tree level is significant since, it seems difficult to build on the LF a systematic procedure to obtain semiclassical approximation.

It is worth remarking also that we have made an ad hoc choice of only one (of the family) of the characteristic LF hyperplanes, $x^{+}=$const., in order to quantize the theory. The conclusions here confirm the conjecture [7] made earlier on the irrelevance in the quantized theory of the fact that the hyperplanes $x^{ \pm}=0$ constitute characteristic 
surfaces of hyperbolic partial differential equation. The discussion given in this paper may clearly be repeated for the case of the non-covariant gauges such as $\partial_{-} A_{-}=0$.

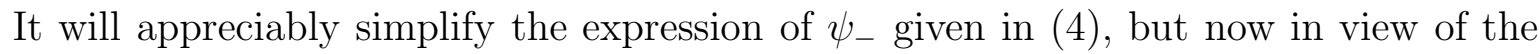
form of the gauge field propagator, we will require the well known prescriptions [27] in order to recover on the LF the conventional covariant theory results.

\section{Acknowledgments}

We acknowledge with thanks the helpful comments from Richard Blankenbecler, Sidney Drell, Michael Peskin, and Gerald Miller during the progress of the work. The hospitality offered to PPS at the SLAC and a financial grant from the Prociência program of the UERJ, Rio de Janeiro, Brazil, are gratefully acknowledged.

\section{References}

[1] P.A.M. Dirac, Rev. Mod. Phys. 21, 392 (1949).

[2] S.J. Brodsky, Light-Cone Quantized QCD and Novel Hadron Phenomenology, SLAC-PUB-7645, 1997; S.J. Brodsky and H.C. Pauli, Light-Cone Quantization and QCD, Lecture Notes in Physics, vol. 396, eds., H. Mitter et. al., SpringerVerlag, Berlin, 1991; S.J. Brodsky, H. Pauli and S.S. Pinsky, Phys. Rept. 301, 299 (1998).

[3] K.G. Wilson et. al., Phys. Rev. D49, 6720 (1994); K.G. Wilson, Nucl. Phys. B (proc. Suppl.) 17, (1990); R.J. Perry, A. Harindranath, and K.G. Wilson, Phys. Rev. Lett. 65, 2959 (1990).

[4] P.P. Srivastava, Light-front Quantization of Field Theory: Some New Results, Lectures at the IX Brazilian School of Cosmology and Gravitation, July 1998, Rio de Janeiro, to be published in the Proceedings, Ed. M. Novello, preprint CBPFNF-003/99, hep-th/9901024; Nuovo Cimento A 107, 549 (1994). See [2,3,4] for the earlier references.

[5] D. Bigatti and L. Susskind, Review of matrix theory, hep-th/9712072; Phys. Lett. B425, 351 (1998), hep-th/9711063.

[6] E. Witten, Commun. Math. Phys.92, 455 (1984). 
[7] P.P. Srivastava, Phys. Letts. B 448, 68 (1999), hep-th/9811225; Mod. Phys. Letts. A 13, 1223 (1998); hep-th/9610149.

[8] J.B. Kogut and D.E. Soper, Phys. Rev. D1, 2901 (1970); D.E. Soper, Phys. Rev. D4, 1620 (1971).

[9] T.M. Yan, Phys. Rev. D7, 1760 (1973) and the earlier references contained therein.

[10] J.D. Bjorken, J.B. Kogut and D.E. Soper, Phys. Rev. D3, 1382 (1971).

[11] G.P. Lepage and S.J. Brodsky, Phys. Rev. D22, 2157 (1980).

[12] See, A. Bassetto, G. Nardelli and R. Soldati, Yang-Mills Theories in Algebraic Non-Covariant Gauges, World Scientific, 1991; A. Bassetto and G. Nardelli, Int. J. Mod. Phys. A12, 1075 (1997); hep-th/9609185

[13] R.J. Perry, Light-front quantum chromodynamics, nucl-th/9901080.

[14] P.A.M. Dirac, Lectures in Quantum Mechanics, Belfer Graduate School of Science, Yeshiva University Press, New York, 1964; Can. J. Math. 2, 129 (1950); E.C.G. Sudarshan and N. Mukunda, Classical Dynamics: a modern perspective, Wiley, NY, 1974.

[15] See J.D. Bjorken and S.D. Drell, Relativistic Quantum Fields, McGraw-Hill, 1965.

[16] S.J. Brodsky, R. Roskies and R. Suaya, Phys. Rev. D8, 4574 (1973).

[17] M. Burkardt and A. Langnau, Phys. Rev., D47, 3452 (1993).

[18] G.A. Miller and R. Machleidt, "Light front theory of nuclear matter," nuclth/9811050.

[19] H.C. Pauli and S.J. Brodsky, Phys. Rev. D32, 2001 (1985).

[20] S.J. Brodsky, J.R. Hiller and G. McCartor, hep-ph/9903388; Phys. Rev. D58, 25005 (1998); hep-th/9802120.

[21] P.P. Srivastava, Nuovo Cimento A 108, 35 (1995); hep-th/9412204205.

[22] See, N. Nakanishi and I.Ojima, Covariant Operator Formalism of Gauge Theories and Quantum Gravity, World Scientific, 1990; O. Piguet and S.P. Sorella, Algebraic Renormalization, Springer-Verlag, Berlin, 1995.

[23] C. Becchi, A. Rouet and R. Stora, Ann. Phys. (N.Y.) 98, 287 (1976). 
[24] See, for instance, P.P. Srivastava, in Geometry, Topology and Physics, Apanasov et. al. (Eds.), Walter de Gruyter \& Co., Berlin, New York, 1997, pp. 260; hepth/9610149. The $L S$ Spin operator may be defined by $\mathcal{J}_{3}(p) \equiv-W^{+}(p) / p^{+}$where $W^{\mu}$ is Pauli-Lubanski four vector. We can verify the identity:

$$
\mathcal{J}_{3}(p)=e^{\left(-\frac{i}{p^{+}}\right)\left(B_{1} p^{1}+B_{2} p^{2}\right)} J_{3} e^{\left(\frac{i}{p^{+}}\right)\left(B_{1} p^{1}+B_{2} p^{2}\right)}=J_{3}-\frac{1}{p^{+}}\left(p^{1} B_{2}-p^{2} B_{1}\right)
$$

where $\sqrt{2} B_{1}=\left(K_{1}+J_{2}\right)$ and $\sqrt{2} B_{2}=\left(K_{2}-J_{1}\right)$ are the kinemetical boost operators in the standard notation. For the Dirac spinor we obtain

$$
\mathcal{J}_{3}(p)=\frac{1}{2}\left[I+\frac{\left(\gamma^{\perp} p_{\perp}\right) \gamma^{+}}{p^{+}}\right] \Sigma_{3}
$$

with the property $\mathcal{J}_{3}(p) u^{(r)}(p)=(r / 2) u^{(r)}(p)$ where $r= \pm$. The other dynamical components $\mathcal{J}_{1.2}(p)$ may also be defined.

[25] P. P. Srivastava and E.C.G. Sudarshan, Phys. Rev. 110, 765 (1958). In fact, in LF coordinates we have $\int d^{4} p \theta\left( \pm p^{+}\right) \theta\left( \pm p^{-}\right) \delta\left(p^{2}-m^{2}\right)=$

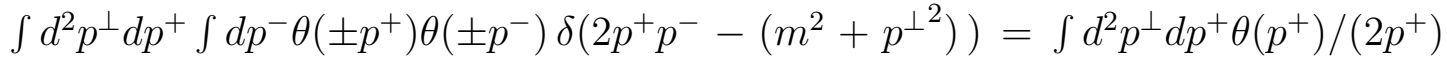
analogous to the conventional one where $\int d^{4} p \theta\left( \pm p^{0}\right) \delta\left(p^{2}-m^{2}\right)=\int d^{3} \vec{p} /\left(2 E_{p}\right)$ with $E_{p}=+\sqrt{\vec{p}^{2}+m^{2}}>0$.

[26] The existence of the continuum (or infinite volume) limit of DLCQ was demonstrated in P.P. Srivastava, Light-front quantization and Spontaneous Symmetry Breaking-Discretized formulation, Hadron Physics 94, p. 253, Eds. V. Herscovitz et. al., World Scientific, Singapore, 1995; hep-th/9412204, 205.

[27] S. Mandelstam, Nucl. Phys. B213, 149 (1983); G. Leibbrandt, Phys. Rev. D29, 1699 (1984). 